NBER WORKING PAPER SERIES

THE EFFECT OF RISK ON THE FIRM'S

OPTIMAL CAPITAL STOCK: A NOTE

Kevin J. Maloney

William J. Marshall

Jess B. Yawitz

Working Paper No. 1132

NATIONAL BUREAU OF ECONOMIC RESEARCH

1050 Massachusetts Avenue

Cambridge MA 02138

May 1983

The authors wish to acknowledge helpful comments from David Baron, Thomas Gilligan, Edward Greenberg, Ira Horowitz, James Little, and an anonymous referee. The research reported here is part of the NBER's research program in Financial Markets and Monetary Economics. Any opinions expressed are those of the authors and not those of the National Bureau of Economic Research. 


\section{The Effect of Risk on the Firm's Optimal Capital Stock: A Note}

by

Kevin J. Maloney William J. Marsha 11 Jess B. Yawitz

\section{ABSTRACT}

In this paper we extend the recent work on the choice of input mix under uncertainty. In particular, we demonstrate that the qualitative nature of the disturbance term, along with the decision sequence, is a crucial determinant of the overall effect of uncertainty on the optimal input mix of a firm. Using general demand and production functions in conjunction with a mean-variance framework for financial valuation, we demonstrate the differential effects of systematic and non-systematic risk on the firm's choice of an optimal input mix. Consistent with earlier work in economics, this analysis demonstrates that uncertainty, regardless of the source, has important implications for the firm's choice of technology.

Institute of Banking and Financial Markets School of Business Administration Washington University

St. Louis, Missouri 63130

(314) $889-6340$ 


\section{Introduction}

Much of the recent work in financial economics can be viewed as an attempt to synthesize contemporary financial market theory with the theory of the firm under uncertainty. This line of research analyzes firm behavior using models that explicitly incorporate the capital market's valuation of risk. The early results indicate that this basic approach holds great promise in its ability to consider the financial market effects on a host of decisions made at the firm level.

In this paper we extend the recent work of Greenberg, Marshall, and Yawitz [1] and Shrieves [3] on the choice of input mix under uncertainty. In particular, we demonstrate that the qualitative nature of the disturbance term, along with the decision sequence, is a crucial determinant of the overall effect of uncertainty on the optimal input mix of a firm. Using general demand and production functions in conjunction with a mean-variance framework for financial valuation, we demonstrate the differential effects of systematic and non-systematic risk on the firm's choice of an optimal input mix. Consistent with earlier work in economics, this analysis demonstrates that uncertainty, regardless of the source, has important implications for the firm's choice of technology. ' When the source of the uncertainty is firm specific, there is no need to take account of any reaction in the capital markets; the risk-free rate of interest is the only relevant financial market variable. We refer to the relationship between this type of uncertainty and the choice of an optimal input mix as the "technical effect." However, when the uncertainty is of the type that affects all firms in the economy, the technology decision must also explicitly recognize the reaction of the financial markets. In a rate of return framework, this market feedback will serve to alter the firm's (or project's) required capitalization rate. This "finan- 
cial effect" will, for the majority of firms, partially reverse the technical effect.

\section{Sources of Risk and the Optimal Capital Stock}

In this paper we analyze the input choices of a firm that faces uncertain demand for its product. The model is attractive in that it captures two very important features of firm behavior. First, the uncertainty is the result of demand disturbances. This specification seems reasonable since the firm presumably has more information about technological and cost factors than it has about consumer behavior. Second, the model affords the firm flexibility in its production decision. In particular, we assume that the firm is allowed to choose its variable factor inputs after demand is revealed.

The particular model used in this paper assumes that the firm produces its output using capital $(K)$ and labor (L) according to the following production function.

$$
\text { (1) } q=F(K, L) \quad F_{K}, F_{L}>0 \quad F_{K K}, F_{L L}<0
$$

The choice of output and input levels proceeds in two stages. Prior to the revelation of the actual demand for $i$ ts product, but with the knowledge of the distribution of demand, the firm must choose its capital stock. The demand curve is then revealed and the firm selects the quantity of labor (and consequently the price and quantity of its output) that maximizes profit. $^{2}$ The only decision made under uncertainty (ex ante) is the choice of $K$. This choice is made so as to maximize the net present value of the cash flows. Under the assumptions of the Capital Asset Pricing Model (CAPM), the value of the cash flows is given by equation (2). 
(2) $v_{i}=\frac{1}{R_{F}}\left[E\left(x_{i}\right)-\lambda \operatorname{cov}\left(x_{i}, x_{M}\right)\right]$

$v_{i}=$ value of the firm

$R_{F}=$ one plus the risk free rate of interest

$x_{i}=$ net cash flow of firm $i$

$x_{m}=$ net cash flow to the market portfolio

$\lambda=$ the market price of risk.

The net cash flow is defined as revenue minus the variable costs of production. The net present value of these cash flows is simply $v_{i}$ minus the acquisition cost of the capital, where $\mu$ is the price of capital goods. 3

(3) $N P V_{i}=v_{i}-\mu K$

From this perspective, it is clear that the cash flow variable in equation (2) must incorporate this conditional optimization. To analyze the effects of demand uncertainty we assume that the firm faces an inverse demand function of the form

$$
\text { (4) } p=p(q)+\varepsilon, 4
$$

where $\varepsilon$ has zero mean, and $\varepsilon$ and $X_{M}$ are jointly normal variables with variances $\sigma_{\varepsilon}^{2}$ and $\sigma_{M}^{2}$, respectively. Defining $q^{*}(K, \varepsilon)$ as the optimal conditional output, $X_{i}$ is given by

$$
\text { (5) } x_{i}=\left[p\left(q^{*}(K, \varepsilon)\right)+\varepsilon\right] q^{*}(K, \varepsilon)-c\left(q^{*}(K, \varepsilon), K\right)=x_{i}^{*}(K, \varepsilon) \text {, }
$$

where $C\left(q^{*}(K, \varepsilon), K\right)$, the variable cost of production, is an increasing convex function of $q$ with $\frac{\partial^{2} c}{\partial q \partial K}<0$. From Hoelling's Lemma it follows that 
$X_{i}^{*}(K, \varepsilon)$ is an increasing function of $\varepsilon$. Using this relationship, we are able to determine the effect of uncertainty on the choice of an optimal capital stock.

Substituting equations (2) and (5) into equation (3), we obtain

(6) $N P V_{i}=\frac{1}{R_{F}}\left[E\left[X_{i}^{*}(K, \varepsilon)\right]-\lambda \operatorname{cov}\left[X_{i}^{*}(K, \varepsilon), X_{M}\right]\right]-\mu K$.

Note that $\operatorname{cov}\left(X_{i}^{*}(K, \varepsilon), X_{M}\right)$ will be non-zero only if $\varepsilon$ is correlated with $X_{M}$. Thus, there are valuation effects associated with systematic disturbances that are not associated with firm specific disturbances. 5 The implication of this distinction for the choice of an optimal capital stock will now be analyzed.

The first order condition for the maximization of net present value is given by

(7) $\frac{\partial N P V_{i}}{\partial K}=\frac{1}{R_{F}}\left[E\left[\frac{\partial X_{i}^{*}(K, \varepsilon)}{\partial K}\right]-\lambda \operatorname{cov}\left[\frac{\partial x_{i}^{*}(K, \varepsilon)}{\partial K}, X_{M}\right]\right]-\mu=0$. $\operatorname{cov}\left[\frac{\partial x_{i}^{*}(K, \varepsilon)}{\partial K}, x_{M}\right]$, the covariance between the incremental cash flows and the cash flows to the market portfolio, will be of the same sign as $\operatorname{cov}\left(\varepsilon, X_{M}\right)$, except if the demand disturbance is firm specific in which case this term vanishes. ${ }^{6}$ In that case the optimal capital stock, $K^{*}$, is defined implicitly by (8) $\frac{1}{R_{F}} E\left[\frac{\partial X_{i}^{*}(K, \varepsilon)}{\partial K}\right]-\mu=0$.

Note that $K^{*}$ is also the solution that would obtain if risk neutrality were assumed, since in a risk neutral world $\lambda=0.7$

Since $\lambda>0$ the optimal capital stock in presence of systematic risk, $K^{* * *}$, will be lower (higher) than $k^{*}$ if $\operatorname{cov}\left(\varepsilon, x_{M}\right)>0(<0)$. This follows because the covarinace of the incremental cash flows and $X_{M}$ has the same 
sign as $\operatorname{cov}\left(\varepsilon, x_{M}\right)$, and because the second order condition for yalue maximization implies that NPV must be concave in $K$.

The intuition here is straightforward. Holding constant total demand variability, an increase in the systematic component will reduce the optimal capital stock since "flexibility" in the production process becomes increasingly important. By replacing fixed costs (capital) with variable costs (labor), the firm is able to dampen the procyclical nature of its profit stream, and thereby reduce the penalty for positive coyariance. At the same time this reduces expected profits. This substitution of labor for capital continues to the point where the valuation effect from an additional reduction in profit variability is exactly equal to the yaluation effect from an additional decrease in expected profit.

Given the above results, one can also demonstrate that $\frac{d k^{*}}{d \lambda}<0$. for the positive covariance firm, indicating that further substitution of labor for capital is justified for the reasons cited above.

We have demonstrated that a systematic demand disturbance leads to a lower optimal capital stock than does an equal size non-systematic disturbance. We emphasize that this does not imply that non-systematic risk has no effect on the capital stock decision of a firm. To the contrary, there are technical effects that accompany both systematic and non-systematic risk.

An increase in the yariance of $\varepsilon$, whether systematic or non-systematic will affect the optimal capital stock, independent of any financial effect. Equivalently, even in a risk neutral world the optimal capital stock of a firm will depend upon the variance of demand. The basic argument is as follows. Since marginal costs are a positive function of output, $x_{i}^{*}(k, \varepsilon)$ is convex in $\varepsilon$ for any given level of $K^{8}$ Thus, the expected value of $x_{i}^{*}(K, \varepsilon)$, 
and therefore expected profit, is a decreasing function of the variance of $\varepsilon$. If it is possible to alter the convexity of $x_{i}^{*}(k, \varepsilon)$ by adjusting $k$, then the firm will have an incentive to change $K$ when the variance of $\varepsilon$ changes. Stated alternatively, if substitution of labor for capital flattens the firm's marginal cost curve, it will also change the sensitivity of expected profits to the variance in demand. Given the general nature of the demand and cost function assumed, it is impossible to determine whether an increase in $\sigma_{\varepsilon}^{2}$ induces the firm to choose a higher or lower capital stock. A positive technical effect $\left(\frac{d K^{*}}{d \sigma^{2}}>0\right)$ will occur if two conditions are met. First, expected profits must be concave in K. This will be met if the second order sufficiency condition for expected profit maximiation is satisfied. Second, the convexity of the relationship between $x_{i}^{*}$ and $\varepsilon$ must be increasing in $k^{9}$ It is this latter condition that cannot be signed for general demand and cost functions. However, it is quite reasonable to believe that an increase in $K$ will flatten the marginal cost curve for most cost functions. This in conjunction with a marginal revenue curve that is linear or convex is sufficient to ensure that the technical effect is positive. 10

\section{Conclusions}

We have demonstrated that even under risk neutrality demand uncertainty will affect the optimal capital stock. We term this the technical effect and have shown it is present regardless of whether the risk is systematic or non-systematic in nature. We have also demonstrated that systematic risk causes the typical (positive covariance) value maximizing firms to choose a smaller capital stock than would be selected if only the technical effect were considered. That is, for firms whose income is positively correlated 
with the market as a whole, the financial effect induces a decreased demand for capital in an amount depending on the size of the covariance between the incremental cash flow to the firm from a given increase in capital and the returns to the market portfolio. 
In this Appendix we prove formally that a sufficient condition for a positive technical effect is that the convexity of $X_{i}^{*}(K, \varepsilon)$ in $\varepsilon$ must be increasing in $K$. Let $f(K, \varepsilon)=\frac{\partial x_{i}^{*}(K, \varepsilon)}{\partial K}$, and let $\hat{K}$ be the optimal capital stock when $\varepsilon=0$ with certainty. By Taylor series expansion $f(K, \varepsilon)$ can be expressed as follows

$$
\text { (A) } f(K, \varepsilon)=f(\hat{K}, 0)+\varepsilon \frac{\partial f(K, \varepsilon)}{\partial \varepsilon}+(K-\hat{K}) \frac{\partial f(K, \varepsilon)}{\partial K} \text {, }
$$

where the derivatives are evaluated at $\hat{\varepsilon}=\alpha \varepsilon$ and $\hat{K}=\beta K+(1-\beta) \hat{K}$ for $0<\alpha, \beta<1$. An expected profit maximizer will set the expected value of (Al) equal to $\mu R_{F} \cdot 11$ By construction $f(\hat{K}, 0)$ will also equal $\mu R_{F}$. Therefore, the optimal capital stock of an expected profit maximizer can be related to $\hat{K}$ as follows

$$
\text { (A2) } K=\hat{K}-\frac{E\left[\varepsilon \frac{\partial f(K, \varepsilon)}{\partial \varepsilon}\right]}{E\left[\frac{\partial f(K, \varepsilon)}{\partial K}\right]}=\hat{K}-\frac{\sigma_{\varepsilon}^{2} E\left[\frac{\partial^{2} f(K, \varepsilon)}{\partial \varepsilon^{2}}\right]}{E\left[\frac{\partial f(K, \varepsilon)}{\partial K}\right]} \text {, }
$$

since $\varepsilon$ is normally distributed. The numerator of (A2) is negative from the second order condition for expected profit maximization. Therefore, the technical effect will be positive $(K>\hat{K})$ if $E\left[\frac{\partial^{2} f(K, \varepsilon)}{\partial \varepsilon^{2}}\right]>0$. But this is precisely the condition reported in the text and in footnote 10 . 
covariance of incremental cash flows and the cash flows of the market will depend on the sign of $\frac{\partial q^{*}(K, \varepsilon)}{\partial K}$. Since $q^{*}$ is defined implicitly by the condition that marginal revenue (MR) equal marginal cost (MC), this derivative must be positive.

$$
\frac{\partial q^{*}(K, \varepsilon)}{\partial K}=\frac{\left(\frac{d M C}{d K}\right)}{\left(\frac{d M R}{d q}-\frac{d M C}{d q}\right)}>0
$$

Thus, for our representative firm $\operatorname{cov}\left(\frac{\partial x_{i}^{*}}{\partial K}, x_{M}\right)$ is positive.

7 Since $k^{*}$ is the solution that obtains when systematic risk is not present, one would expect the same solution for an expected profit maximizer. This is indeed the case. Multiplying equation (8) through by $R_{F}$, we obtain a condition that states that the marginal contribution of a unit of capital to the expected value of revenues minus labor costs must equal the implicit rental rate of capital. This is precisely the condition one obtains when maximizing expected profits.

${ }^{8}$ From Hotelling's Lemma we know that $\frac{\partial X_{i}^{*}(K, \varepsilon)}{\partial \varepsilon}=q^{*}(K, \varepsilon)$. Since $q^{*}$ is defined implicitly by the condition that $M R=M C$, the derivative of $q^{*}$ with respect to $\varepsilon$ is given by

$$
\text { (F 3) } \frac{\partial q^{*}(K, \varepsilon)}{\partial \varepsilon}=-\frac{\left(\frac{d M R}{d \varepsilon}\right)}{\left(\frac{d M R}{d q}-\frac{d M C}{d q}\right)}=\frac{-1}{\left(\frac{d M R}{d q}-\frac{d M C}{d q}\right)}>0
$$

Thus, $x_{i}^{*}(K, \varepsilon)$ is a convex function of $\varepsilon$.

9. A formal proof of this is given in the Appendix.

10 As demonstrated in the Appendix, whether the convexity of the relationship between $x_{i}^{*}$ and $\varepsilon$ is a positive or negative function of $k$ hinges on the the sign of $\frac{\partial^{3} x_{i}^{*}(K, \varepsilon)}{\partial \varepsilon^{2} \partial K}=\frac{\partial^{2} q^{*}(K, \varepsilon)}{\partial \varepsilon \partial K}$. Differentiating the expression for $\frac{\partial q^{*}(K, \varepsilon)}{\partial \varepsilon}$ from footnote (8). with respect to $k$, we obtain 


\section{Footnotes}

'While this paper analyzes the choice of an optimal stock of physical capital, our approach is generalizable to other decisions including advertising expenditure, research and development, etc.

2The model is sufficiently general to include both competitive and noncompetitive structures in the output market. However, we assume that the input markets are competitive. Where appropriate, we will discuss the influence of product market structure on the results of the paper.

3 since we are using an explicit valuation framework, the input price of capital need not include a capitalization rate.

4. The assumption of additive uncertainty is made simply for convenience. The qualitative results remain if the uncertainty is multiplicative.

This statement needs to be qualified slightly. Since this firm is a part of the market portfolio, even firm specific disturbances will appear in $X_{M}$. However, given the large number of firms in the economy, this effect will be of such a small magnitude for the average firm in the capital market that it can be essentially ignored. In any event, it is clear that the covariance between the cash flows of a particular firm and the market is usually dominated by system wide disturbances.

${ }^{6}$ The covariance between the incremental cash flows to this firm and the cash flow to the market portfolio will be positive if $\varepsilon$ and $x_{M}$ are positively correlated. The following argument demonstrates this point. Since $\varepsilon$ and $X$ are joint normal variables,

$$
\text { (Fil) } \operatorname{cov}\left(X_{i}^{*}(K, \varepsilon), X_{M}\right)=E\left[\frac{\partial X_{i}^{*}(K, \varepsilon)}{\partial \varepsilon}\right] \operatorname{cov}\left(\varepsilon, X_{M}\right)=E\left[q^{*}(K, \varepsilon)\right] \operatorname{cov}\left(\varepsilon, X_{M}\right) \text {. }
$$
gince $\frac{\partial X_{i}^{*}(K, \varepsilon)}{\partial \varepsilon}=q^{*}(K, \varepsilon)$ by Hotelling's Lemma. Therefore, the sign of the 


$$
(F 4) \frac{\partial^{2} q^{*}(K, \varepsilon)}{\partial \varepsilon \partial K}=\frac{\left[\frac{d^{2} M R}{d q^{2}} \frac{\partial q^{*}(K, \varepsilon)}{\partial K}-\frac{d^{2} M C}{d \bar{q} d K}\right]}{\left[\frac{d M R}{d q}-\frac{d M C}{d q}\right]^{2}}
$$

Since $\frac{\partial q^{*}(K, \varepsilon)}{\partial \varepsilon \partial K}$ is positive, a non-negative second derivative for the marginal revenue curve and a marginal cost curve that is flatter the higher $K$ are sufficient to make this derivative positive, and yield a positive technical effect.

11 Loistl (1976) discusses the conditions under which this is a valid procedure. Basically, the requirements are that the partial derivatives of $f(K, \varepsilon)$ between $K$ and $\hat{K}$, and between $\varepsilon$ and 0 must $a l l$ exist and be continuous. 


\section{References}

(1) E. Greenberg, W. Marshall, and J. Yawitz, "The Technology of Risk and Return." American Economic Review, June 1978, 241-51.

(2) 0. Loistl, "The Erroneous Approximation of Expected Utility by Means of a Taylor's Series Expansion: Analytic and Computational Results." American Economic Review, December 1976, 904-10.

(3) R. Shrieves, "Uncertainty, the Theory of Production, and Optimal Operating Leverage." Southern Economic Journal. January 1981, 690-702. 